\title{
PHASES OF DIASTOLE IN VARIOUS SYNDROMES OF MITRAL VALVULAR DISEASE
}

\author{
BY \\ P. G. F. NIXON AND G. H. WOOLER \\ From the Departments of Thoracic Surgery and Medicine, The General Infirmary, Leeds
}

Received September 24, 1962

The diastolic events of the left side of the heart are modified by the obstruction of mitral stenosis and the disordered heart volumes of mitral incompetence, and tracings that illustrate the abnormalities have great diagnostic value. Methods developed recently for measuring pressure and movement simultaneously with the heart sounds have allowed records of the patterns of diastolic left heart activity to supplement the physical examination in clinical practice. A degree of confusion has been caused by the use of similar terms in different contexts, and by the failure to recognize the occurrence, significance, or coincidence of certain events. This paper describes the phases of diastole and the syndromes of mitral valvular disease that were observed in the ward and the laboratory, and discusses their bearing upon the genesis of the third heart sound.

\section{SubJECTS AND METHODS}

Patients with chronic rheumatic heart disease were selected for this study when it was considered that the mitral valvular lesion was the sole or the predominant cause of symptoms and signs. Most had been referred for pre-operative assessment, and presented with atrial fibrillation and pulmonary hypertension. The phonocardiogram was recorded in every case. In nearly 200 the left atrial pressure pulse was the reference tracing. In 34 the heart sounds were recorded simultaneously with the left atrial and the left ventricular pressure pulses at cardiac catheterization. The displacement curve of the left ventricular apex (see below) was recorded with the heart sounds in more than 100 patients, and with the left atrial pressure pulse and phonocardiogram in $\mathbf{4 0}$ patients. The relation of events described in this study summarizes the experience of the one person who has recorded the tracings. The difficulties in attempting to recognize the synchronous events in records of different phenomena have been discussed by Wiggers (1921).

Displacement Curve of Left Ventricular Apex (A.D.C.). The pulsation of the apex of the left ventricle was recorded by the method of Schneider and Klunhaar (1961). A point on the skin overlying the apex of the left ventricle was selected, and its movement or displacement in a plane perpendicular to the chest wall was described in a linear fashion on the tracing. Outward movement was represented by an upstroke. The sensitivity and the application of the instrument were adjusted to write the diastolic events with great amplification, and the systolic peaks were not reliably recorded in the majority of cases. The apex of the left ventricle was recognized by Mackenzie's (1902) criteria, and the records show the pattern of events that he described.

In the normal subject the first diastolic event to be recorded is the rapid filling wave rising from the mitral valve opening point ( $\mathrm{O}$ in Fig. 1) to the third heart sound point (3P in Fig. 1). From the point 3P the upstroke continues less steeply in the slow filling wave and flattens out in the period of stasis. The wave ' $a$ ' results from atrial activity, and may be very small if it occurs in a period of stasis. When a third sound is present it occurs synchronously with the third sound point and, from the peak of a steep rapid filling wave, the apex of the left ventricle undergoes a brief inward movement (Fig. 1B) (Dock, Grandell, and Taubman, 1955). When diastole is too short for stasis to occur the A.D.C. ' $a$ ' wave may be more prominent, and low frequency fourth heart sound vibrations may coincide with its apex (Fig. 1B).

The Phonocardiogram. High or medium-frequency phonocardiograms (Leatham, 1952) were recorded in the pulmonary and mitral areas. The mitral area microphone could not be placed in the most 


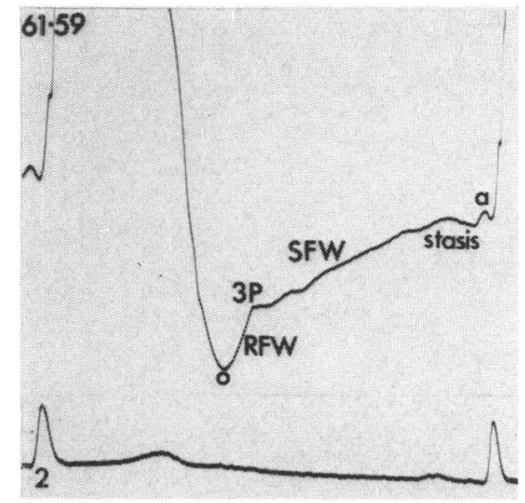

A

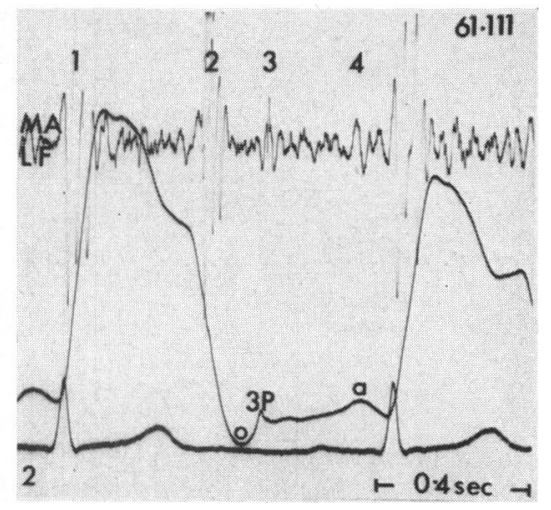

B

FIG. 1(A) and (B).-Displacement curves of the left ventricular apex from two normal adolescents. See footnote for symbols and abbreviations used in the figures.*

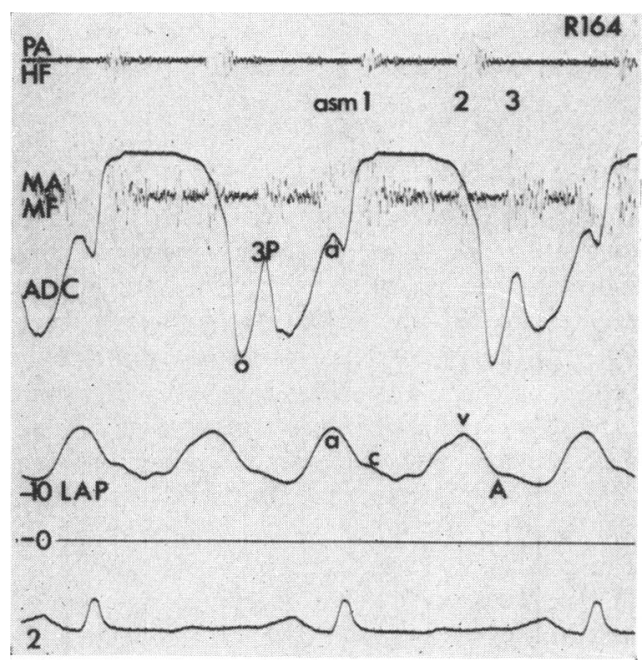

Fig. 2.-Severe mitral incompetence with sinus rhythm. An atrial systolic murmur (asm) is present.

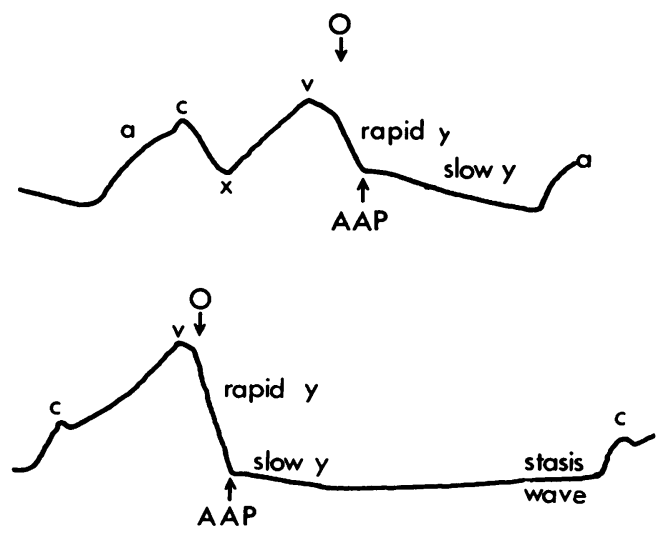

FIG. 3.-Tracings of left atrial pressure pulses. Upper figure: mitral obstruction with regurgitation in sinus rhythm; lower figure: mitral regurgitation with atrial fibrillation.

* Abbreviations and symbols used in the figures.

$2=$ the lead II electrocardiogram.

Phonocardiograms: PA, MA = pulmonary and mitral areas; HF, MF, and LF =high, medium, and low frequency; $1,2,3,4, \mathbf{O}=$ first, second, third, and fourth heart sounds and mitral opening snap; asm=atrial systolic murmur; TM = tricuspid and mitral valve closure transients; $\mathrm{SM}, \mathrm{DM}=$ mitral systolic and diastolic murmurs.

Displacement curves of the left ventricular apex (ADC):

$\mathbf{0}$-atrio-ventricular valve opening point or the beginning of the rapid-filling wave (RFW); $3 \mathbf{P}=$ third sound point or the end of the rapid-filling wave; $S F W=$ slow filling wave; $a=$ displacement resulting from atrial contraction.

Pressure pulses: LA or LAP, LV, BA =left atrial, left ventricular, and brachial arterial; $a, c, v, s w=$ left atrial ' $a$ ', ' $c$ ', ' $v$ '. and stasis waves; $x, y=l e f t$ atrial ' $x$ ' and ' $y$ ' descents; $A$ or $\mathbf{A A P}=$ left atrial annular ascent point. 
advantageous site for recording the third heart sound and mitral diastolic murmur when the skin overlying the apex of the left ventricle was covered by the displacement curve transducer, or transfixed during needle puncture of the left ventricle. The phonocardiogram was examined with special reference to the vibrations of aortic valve closure, the mitral opening snap, the third heart sound, and the mitral diastolic murmur. Aortic valve closure was considered to cause the first vibrations of the second heart sound in the pulmonary area. The opening snap and third sound were identified by their relation to each other and to the reference tracings. The vibrations of the opening snap usually had components of higher frequency than the third heart sound, and could often be recorded in the pulmonary area. A third heart sound was considered to be present when a transient occurred synchronously with the third sound point of the A.D.C., or the annular ascent point of the left atrial pulse, and measured more than twice the size of adjacent diastolic murmur vibrations.

Atrial systolic murmurs were recognized by their occurrence at the time of the ' $a$ ' wave of the A.D.C. or left atrial pulse. They were recorded in severe mitral regurgitation as well as in mitral stenosis when sinus rhythm was present (Fig. 2).

The Left Atrial Pulse. This was obtained by the Ross (1959) technique for transeptal left heart catheterization in the manner described previously (Nixon, 1960). The patient was supine, the zero reference point was the sternal angle, and the pressure pulses were calibrated in $\mathrm{mm}$. $\mathrm{Hg}$. The tracings of the left atrial pulses illustrated in Fig. 3 show the landmarks that were recognized.

In sinus rhythm the pressure rose in the ' $a$ ' wave of atrial activity and declined in the ' $x$ ' descent. The ' $x$ ' descent appeared to be caused by atrial relaxation (Nixon and Polis, 1962). The 'c' wave, attributable to the bulging of the mitral leaflets in early systole (Braunwald et al., 1955), occurred on the downslope of 'a', at the nadir of ' $x$ ', or on the upstroke to ' $v$ ', according to the duration of the P-R interval (Nixon and Polis, 1962). In atrial fibrillation ' $a$ ' waves and ' $x$ ' descents did not occur. During ventricular systole the pressure rose in the ' $v$ ' wave. The ' $v$ ' peak was recorded during the period of isovolumetric relaxation, 0.01 to 0.04 second after aortic valve closure, and 0.01 to 0.1 second before the mitral opening snap. From the ' $v$ ' peak pressure declined in the ' $y$ ' descent. The most rapid part of the ' $y$ ' descent usually began 0.01 to 0.02 second before the opening snap and invariably ended at the annular ascent point. At the annular ascent point the rate of ' $y$ ' descent slowed, and a brief positive wave, the annular ascent wing, was often recorded (Radner, 1957; Nixon, 1961c). The slow part of the ' $y$ ' descent extended from the annular ascent point or wing to the next positive wave: to the ' $a$ ' wave in sinus rhythm, to the ' $c$ ' wave in atrial fibrillation, or to the stasis wave when the length of diastole and the size of the mitral orifice permitted a period of diastasis (Nixon and Wooler, 1961).

Simultaneous Records of Left Atrial and Ventricular Pressures. These were obtained at cardiac catheterization by puncturing the apex of the left ventricle (Brock, Milstein, and Ross, 1956) when the left atrial catheter was in situ. Equisensitive Statham P23G transducers were used. Experiment showed that the atrial and ventricular pressure recording systems delayed events by an average of 5 and 2.5 milliseconds respectively as compared with the phonocardiographic system.

All sound, A.D.C., and pressure tracings were recorded photographically at $80 \mathrm{~mm}$. $/ \mathrm{sec}$. during expiratory pauses, and a lens and graticule were employed to measure intervals to the nearest $0.1 \mathrm{~mm}$. Sounds were timed from the onset of their vibrations on the phonocardiogram.

Every attempt was made to carry out the investigations in a way that would result in the patient being conscious but comfortable and free from pain and anxiety. Digitalis and sedatives were used, and the patient was made familiar with the laboratory and its staff before cardiac catheterization. These measures allowed records to be taken at slow heart rates.

Surgical Findings. In more than half the patients the mitral valve was examined at valvotomy or open heart operation, and the long diameter of its orifice was determined by palpation.

\section{RESULTS}

The Phases of Diastole. The events recorded in the study of mitral valvular disease divided diastole into a number of phases, namely the phase of isovolumetric left ventricular relaxation, the rapid and the slow left ventricular filling phases, diastasis, and atrial systole.

The beginning of the period of isovolumetric left ventricular relaxation was marked by the sound of aortic valve closure. During the period of isovolumetric left ventricular relaxation the pressure within the left atrium began its decline into the ' $y$ ' descent from the peak of the ' $v$ ' wave, which was 


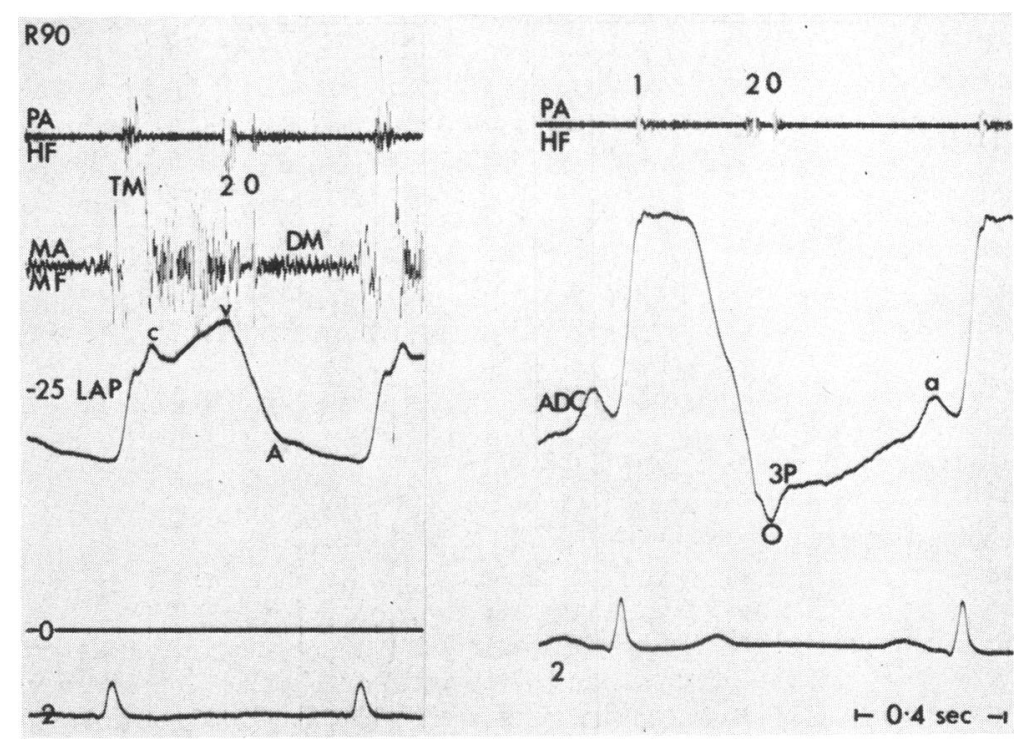

FIG. 4.-Severe mitral obstruction with slight regurgitation.*

recorded 0.01 to 0.04 second after aortic valve closure and 0.01 to 0.1 second before the opening snap (Fig. 2, 4-6).

The phase of isovolumetric left ventricular contraction ended with the opening of the mitral valve. The mitral opening snap vibrations began 0.016 to 0.036 second after the left ventricular pressure fell below the left atrial (Fig. 7). Within 0.02 second of the opening snap the rate of the ' $y$ ' descent often accelerated abruptly into its most rapid phase (Fig. 5-7). The O point of the A.D.C., marking the onset of the rapid filling wave, was recorded at an average interval of 0.025 second after the mitral opening snap (Fig. 4-6).

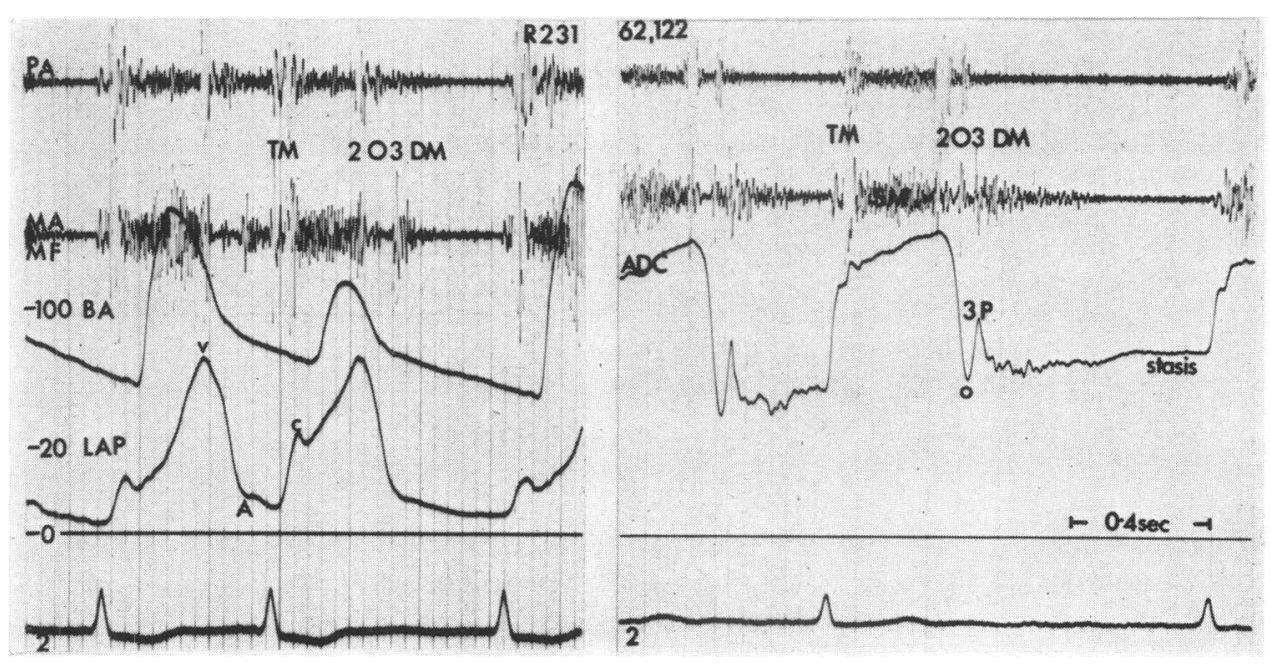

Fig. 5.-Severe mitral incompetence.*

* The key to abbreviations used in these Figures can be found as a footnote on p. 394. 
The phase of rapid left ventricular filling coincided with the most rapid part of the 'y' descent of the left atrial pressure and with the rapid filling wave of the A.D.C. Left ventricular pressure continued to fall, but the rate of decline was very much slowed (Fig. 7). In severe mitral obstruction diastolic murmur vibrations were recorded (Fig. 4). In severe mitral incompetence diastolic murmur vibrations were absent, or were small in relation to their size in the next phase (Fig. 2, 5-7).

The end of the phase of rapid left ventricular filling was marked by a number of events. The diastolic murmur of severe mitral incompetence began, or, if small vibrations had already been present, waxed suddenly loud. The third heart sound was recorded at this time in cases where it was present. The rapid portion of the left atrial ' $y$ ' descent ended at the annular ascent point. The rapid filling wave of the A.D.C. ended at the third sound point and, in mitral obstruction, was succeeded by the more gently inclined ascent of the slow-filling wave (Fig. 2, 4-8). Where third heart sounds were present the rapid filling wave was succeeded by a brief inward movement of the apex of the left ventricle (Dock

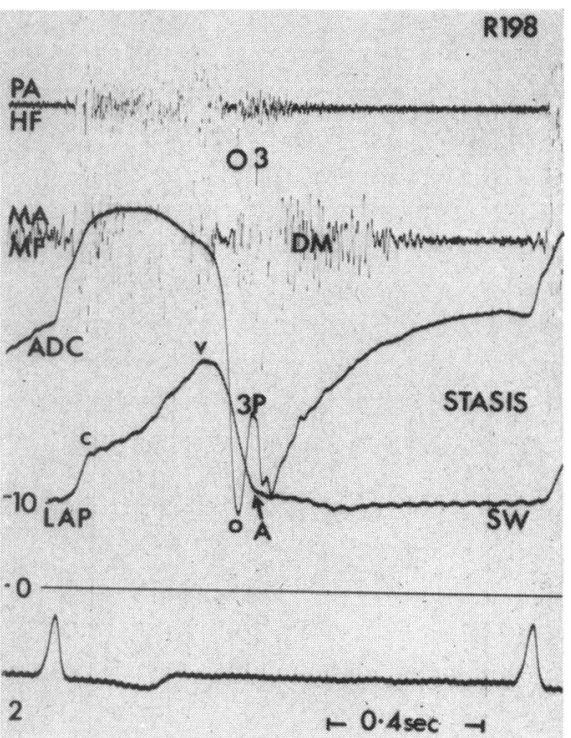

Fig. 6.-Severe mitral incompetence.* et al., 1955; H. A. Snellen and H. Hartman, 1962, personal communication) (Fig. 2, 5, 6). These events occurred together, 0.1 to 0.17 second after aortic valve closure, at a time when the left atrial and ventricular pressures were separated by an abnormally large left ventricular filling pressure gradient in both severe mitral obstruction and severe mitral incompetence (Nixon and Wooler, 1963) (Fig. 7).

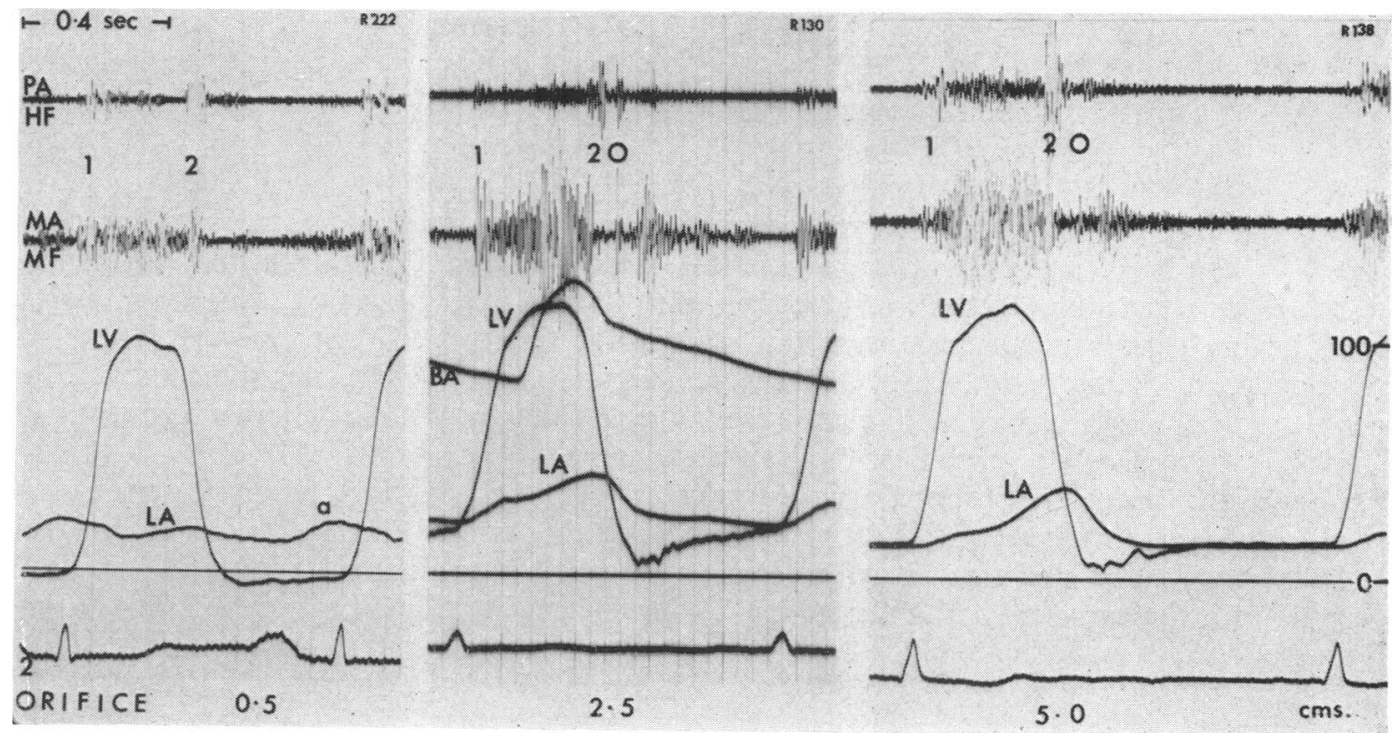

FIG. 7.- Mitral incompetence. Simultaneous left atrial and ventricular pressure pulses from three different patients. In the first the syndrome of severe obstruction was present and the long diameter of the mitral orifice measured $0.5 \mathrm{~cm}$. In the second and third the syndrome of severe mitral incompetence was present and the mitral orifices measured 2.5 and $5.0 \mathrm{~cm}$. respectively.*

* The key to abbreviations used in these Figures can be found as a footnote on p. 394. 
The phase of slow left ventricular filling succeeded the rapid filling phase. Diastolic murmur vibrations were present in mitral stenosis and in severe mitral incompetence, and the left atrial and ventricular pressures were still separated by an abnormally large left ventricular filling pressure gradient. The period coincided with the slow part of the left atrial ' $y$ ' descent, and with the slowfilling wave of the A.D.C. (Fig. 4-8).

In sinus rhythm the slow phase of left ventricular filling ended in atrial systole, and this was marked by the atrial systolic murmur, the 'a' wave of the left atrial pulse, the 'a' wave of the A.D.C., and by an increase in the left ventricular filling pressure gradient (Fig. 2, 8). The authors never saw evidence of stasis in severe mitral disease with sinus rhythm because no recorded diastolic interval was long enough.

In atrial fibrillation the slow filling phase ended in the isovolumetric contraction phase of the next ventricular systole, or in a period of stasis. The duration of the slow filling phase and the manner

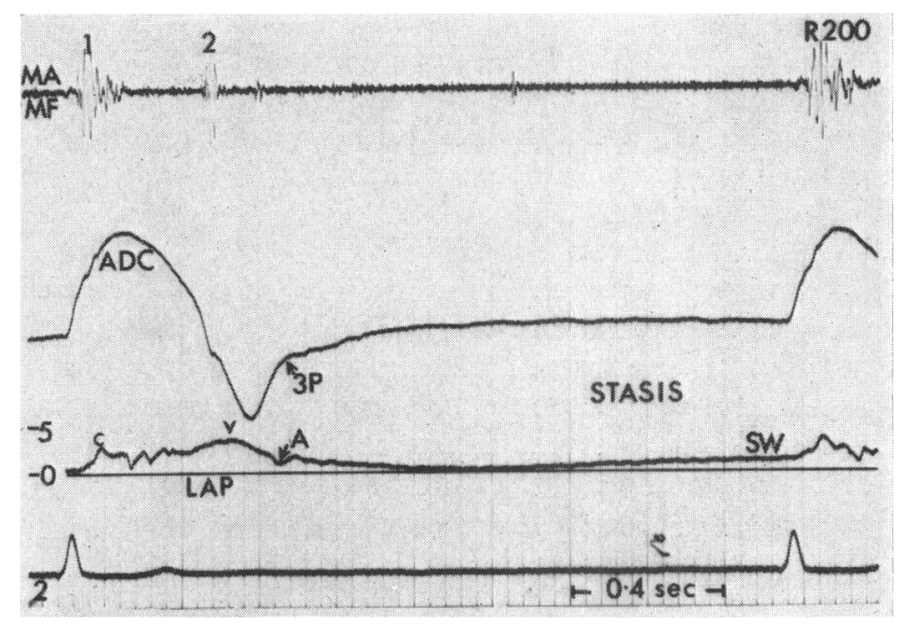

FIG. 8.-Slight mitral obstruction. The mitral orifice is large enough to permit left ventricular filling and stasis.*

of its termination appeared to be determined by the size of the mitral orifice and the duration of diastole. In cases of severe obstruction, where the long diameter of the mitral orifice was found at operation to measure about $1.5 \mathrm{~cm}$. or less, the signs of stasis never appeared, even when diastolic intervals lasting 1.0 second or more were recorded on the tracings. In nearly every case of mild obstruction, or, in incompetence, where the long diameter of the mitral orifice measured about $2.0 \mathrm{~cm}$. or more, the signs of stasis were seen on the records, even though diastolic intervals measuring 0.8 1.0 second or longer were required for their appearance.

The interval between the completion of ventricular filling and the onset of atrial or ventricular systole is known as diastasis. Henderson (1906) created the term to describe this phase of "rest" or stasis, in which the flow of blood into the ventricle is negligible, to distinguish it from the preceding periods of "ventricular relaxation and refilling". Mackenzie (1902), Gibson (1907), and Wiggers (1921) were familiar with the rise in atrial pressure that indicates ventricular stasis: Hirschfelder (1907) named it the ' $h$ ' wave, and Rihl (1904) and Nixon and Wooler (1961) referred to it as the stasis wave. Braunwald et al. (1955) drew attention to the fact that stasis cannot occur in severe mitral stenosis.

In the present study (Fig. 5-8), the left atrial and the left ventricular pressures were equal in diastasis, and no diastolic murmur vibrations were recorded. The left atrial pressure rose in the

* The key to abbreviations used in this Figure can be found as a footnote on p. 394. 
stasis wave which extended from the nadir of the ' $y$ ' descent to the interruption of the next systolic event. The diastolic outward movement of the apex of the left ventricle ceased at the beginning of diastasis, and the slow filling wave of the A.D.C. was replaced by a flat plateau or slow decline.

\section{The Syndromes of Mitral Valvular Disease}

The abnormalities recorded in this study of mitral valvular disease occurred in patterns or syndromes. With very few exceptions the syndromes were discrete, and one component could be predicted when another was recorded. The majority of patients were severely disabled, and the close relation between the syndrome and the mitral lesion made it possible to forecast the anatomical findings before operation. Where the disease was less advanced surgical treatment was not given, and there was little opportunity to correlate the physiological with the anatomical disturbances.

The Syndromes of Mitral Obstruction. The single most important feature of severe mitral obstruction, with or without regurgitation, appeared to be the absence of stasis. The abnormally large left ventricular filling pressure gradient persisted until diastole ended in the atrial or ventricular contraction of the next cardiac cycle. The left ventricular pressure climbed slowly and rarely rose above the sternal angle. The mitral diastolic murmur began with the rapid filling phase and altered little in size before it ended in the atrial systolic murmur or the first heart sound of the next cardiac cycle. Third heart sounds did not occur. The apex of the left ventricle moved outwards during the whole of diastole, and there was no inward movement at the junction of the rapid and slow filling phases of diastole. The junction of the rapid and slow left ventricular filling phases could be recognized in the annular ascent point of the left atrial pulse and in the third sound point of the A.D.C. The syndrome (Fig. 4) occurred in the cases where the long diameter of the mitral orifice measured about $1.5 \mathrm{~cm}$. or less.

Lesser degrees of mitral obstruction permitted the ventricle to fill when the heart rate was slow enough, and the slow filling phase ended in diastasis in the longer diastolic intervals: the abnormally large left ventricular filling pressure gradient disappeared as the left atrial and ventricular pressures equalized, the diastolic murmur faded away, the left atrial ' $y$ ' descent ended in a stasis wave, and the slow filling wave of the A.D.C. flattened out in a plateau. This syndrome occurred in cases where the long diameter of the mitral valve measured 1.5-2.0 cm. or more. Mitral pansystolic murmurs were sometimes recorded, but incompetence was considered to be minimal where third heart sounds did not occur, where left ventricular end-diastolic pressure was not elevated, and where inward movement of the left ventricular apex at the junction of the rapid and the slow ventricular filling phases did not take place (Fig. 8).

One patient with trivial mitral stenosis was investigated. The left atrial 'a' wave was prominent, and an atrial systolic murmur was present, but the records of the rapid and the slow phases of left ventricular filling appeared to be normal.

The Syndromes of Mitral Incompetence. An abnormally large left ventricular filling pressure gradient was present without exception when the left atrial and the left ventricular pressures were measured before operation in severe mitral incompetence. In some patients the incompetence was caused by dilatation of the atrio-ventricular ring, the mitral orifice was larger than normal, and the cusps were not fused. In other patients a degree of adhesion between the mitral cusps had reduced the mitral orifice to not less than about $2.5 \mathrm{~cm}$. It follows that the abnormal mitral diastolic pressure gradient (and the diastolic murmur) cannot be attributed to a degree of stenosis in every case of mitral incompetence (Nixon and Wooler, 1963). Whether or not a degree of adhesion between the cusps was found at operation, the patients with severe mitral regurgitation showed the features of one syndrome (Fig. 5-7).

The rapid phase of left ventricular filling was marked by a steeply climbing rapid-filling wave of the A.D.C., and, commonly, by an unusually steep rapid portion of the ' $y$ ' descent. Diastolic murmur vibrations were absent or small. The junction of the rapid and slow phases of filling was sharply signalled by several events: the onset or the sudden waxing of the diastolic murmur, the third 


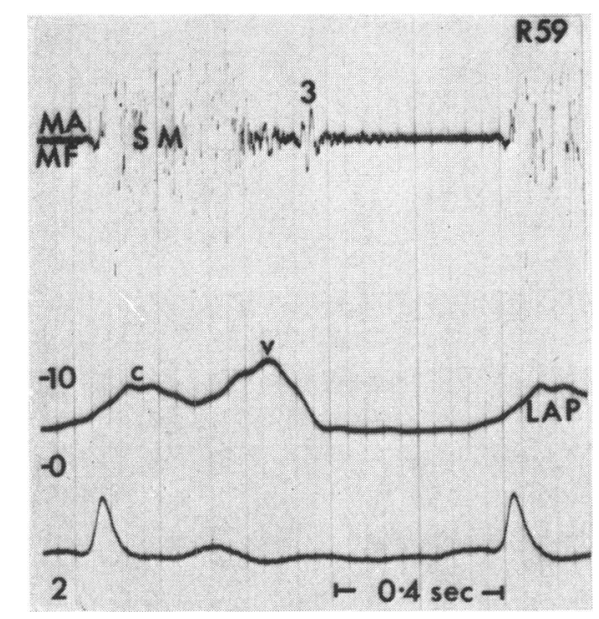

Fig. 9.-Slight mitral incompetence.*

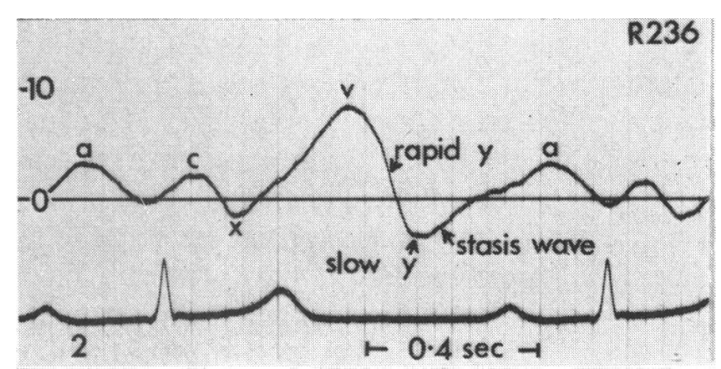

Fig. 10.-The left atrial pressure pulse from a normal young person.*

heart sound, the beginning of a prominent inward movement of the apex of the left ventricle, and the sudden deceleration of the ' $y$ ' descent in the left atrial annular ascent point (Fig. 2, 5-7).

During the slow phase of ventricular filling the left ventricular pressure climbed to abnormally high levels, sometimes to more than $20 \mathrm{~mm}$. $\mathrm{Hg}$, before equalizing with the left atrial pressure at the beginning of diastasis. The diastolic murmur faded away as the abnormally large left ventricular filling pressure gradient disappeared (Fig. 7).

The signs of stasis appeared when the heart rate permitted diastasis: the left atrial and ventricular pressures were equal, the diastolic murmur was absent, the left atrial pressure rose in a stasis wave, and the slow filling wave of the A.D.C. flattened out in a plateau (Fig. 5-7).

In severe mitral incompetence the suppleness and mobility of the aortic leaflet is indicated by the opening snap (Nixon, Wooler, and Radigan, 1960), and a loud opening snap (Fig. 5-7) was the rule rather than the exception in the patients examined by the authors.

When the mitral incompetence appeared to be less severe the elevation of the left atrial and ventricular end-diastolic pressure was smaller, the third sound and diastolic murmur were quieter, and the rapid filling wave of the A.D.C. was less steep. At the end of the rapid filling phase the inward movement of the apex of the left ventricle was less prominent and less easily palpable.

The mitral incompetence was considered to be slightly or moderately severe when the disability did not prevent the patient from working and did not worsen from one season to the next. In such patients the pulmonary arterial pressure and the left ventricular end-diastolic pressure lay close to the normal range. The height of the left atrial ' $v$ ' wave and the rapidity of the ' $y$ ' descent were usually abnormal, but the left atrial stasis wave began soon after the annular ascent point and the phase of slow left ventricular filling was extremely short. Third heart sounds were recorded, and the frequency of their vibrations appeared to be lower than in the severely disabled cases. The opening snap and diastolic murmur had vibrations of low frequency and small amplitude, or were absent (Fig. 9). The rapid filling wave of the A.D.C., and the inward movement of the left ventricular apex at the time of the third sound were not so prominent as in the severely disabled cases. Unlike the severely disabled cases, the duration of systole tended to be normal or shorter than normal.

In trivial mitral incompetence the only abnormal finding was the systolic murmur.

The Syndrome of the Rigid Mitral Valve. About 5 per cent of the severely disabled patients with pulmonary hypertension formed a distinct group. The symptoms were of long duration. Great enlargement of the left atrium and gross calcification of the mitral valve were commonly present.

* The key to abbreviations used in these Figures can be found as a footnote on p. 394. 
The left ventricle was moderately enlarged and a mitral pansystolic murmur was present. The tracings of diastolic events were distinctive in showing features of both severe obstruction and severe incompetence. The mitral diastolic murmur began with the rapid inflow phase but increased considerably in loudness at its junction with the slow inflow phase. The diastolic murmur and the abnormally large left ventricular filling pressure gradient faded away only when diastole lasted for more than about one second. Third sounds occurred infrequently, and opening snaps were absent or small. The apex of the left ventricle moved inwards at the time of the third heart sound, but the movement was slight. The signs of stasis appeared only when diastole lasted for more than about one second.

At operation the mitral valve was found to be immobilized by dense fibrous tissue and calcification: the long diameter of the rigid orifice measured about 1.5 to $2.0 \mathrm{~cm}$., and the obstruction and the regurgitation each appeared to be severe. In cases with small opening snaps the central part of the aortic cusp retained some mobility.

Unexplained Left Ventricular Enlargement and Third Heart Sounds in Mitral Stenosis. In two patients without systolic murmurs the first heart sound was very loud, the diastolic murmur began close to the opening snap and altered little in size as it rumbled through diastole, and the rapid and the slow filling waves of the A.D.C. ascended slowly. These findings suggested simple mitral stenosis, but in each case the systolic thrust of the apex of the left ventricle was abnormally prominent, the A.D.C. showed a small inward movement at the junction of the rapid and the slow filling phases, and a third heart sound was present.

The cause of the left ventricular enlargement and third sound was never found. Either left ventricular infarction from coronary embolism (Oakley, Yusuf, and Hollman, 1961), or an unusual degree of shortening of the chordae tendineae was suspected.

\section{DisCUSSION}

The relations described in this paper are the result of the authors' experience, but the concepts depend to a large extent upon, and appear to be harmonious with, the experiments and observations of Mackenzie (1902), Henderson (1906), Thayer (1909), Hirschfelder (1910), Wiggers (1921), Dock (1949), Dock et al. (1955), Braunwald et al. (1955), Radner (1957), Dock (1959), Rushmer (1961) and Sarnoff, Gilmore, and Mitchell (1962).

The Phases of Diastole. The heart sounds have been related to the diastolic left atrial pressure changes in previous communications (Nixon, 1961a, b, c; Nixon and Wooler, 1961). The relation between the heart sounds, the left atrial pressure cycle, and the diastolic movement of the left ventricular apex is described here. It is shown that the rapid and the slow inflow phases and the period of stasis can be recognized from the A.D.C. as well as from the phonocardiogram and the left atrial pressure tracing.

In the normal heart it appears that the slow filling phase may be brief or non-existent, and stasis may follow closely upon the rapid filling phase (Fig. 10). In severe mitral disease the extent of the large left ventricular filling pressure gradient, the diastolic murmur, the slow part of the left atrial ' $y$ ' descent and the slow filling wave of the A.D.C. constantly indicate the presence of a prominent slow filling phase. In severe mitral obstruction this may be caused by the impediment to rapid left ventricular filling. In severe mitral incompetence, where the mitral orifice may be larger than normal, the prominent slow filling phase may be attributed to the fact that the left atrium still contains an excessive amount of blood when the elongation of the distended ventricle and the ascent of the mitral annulus fibrosus have tautened the cusps and the chordae and reduced the mitral orifice.

Powerful atrial contraction can tauten the mitral valve sufficiently to close the orifice (Sarnoff et al., 1962). The vibrations of the atrial systolic murmur of mitral incompetence begin after the onset of the left atrial and A.D.C. 'a' waves, i.e. after atrial contraction has increased the pressure in the left atrium and pushed outwards the apex of the left ventricle. It seems probable that the atrial contraction increases the left ventricular filling pressure gradient while it reduces the mitral orifice by tensing further the mitral cusps and chordae that are already taut from ventricular distension. 
Diagnosis of Syndromes of Mitral Valvular Disease. In severe mitral obstruction the flow of blood from the left atrium to the left ventricle is impeded, the pressures in the two chambers cannot equalize, and left ventricular stasis cannot occur. In severe mitral incompetence the left atrium holds under high pressure the regurgitated volume in addition to the normal pulmonary venous inflow, and discharges it through a mitral orifice that may be larger than normal; the filling of the left ventricle is manifest in the signs of stasis, and the excessive filling is shown by the raised enddiastolic pressure. Therefore, it is not surprising that a study of the diastolic events should allow the two conditions to be distinguished. Difficulties have arisen when a prolonged slow filling period has been supposed to indicate stenosis.

It has been suggested that severely disabled patients with regurgitation form a continuous spectrum that extends from those with severe stenosis and slight regurgitation to those with severe regurgitation and negligible stenosis. Our cases do not conform to such a pattern. When the long diameter of the mitral orifice measures less than about $1.5 \mathrm{~cm}$. the signs present are those of severe obstruction. When the orifice measures more than about $2.0 \mathrm{~cm}$. the signs are those of severe incompetence, and the aortic leaflet is usually pliant and mobile. A small minority of cases with orifices measuring about $1.5-2.0 \mathrm{~cm}$. show features of obstruction and regurgitation, and their valves are usually rigid.

At present this diagnostic grouping simplifies the choice of surgical treatment. Obstruction is amenable to valvotomy, and severe incompetence may be treated by a form of repair (Wooler et al., 1962). The patients with rigid valves do not appear to be suitable for either operation, and must probably await the introduction of an artificial valve.

The signs of stasis are valuable because they distinguish the lesser degrees of stenosis from the more severe, and the severely incompetent cases from the seriously obstructed: seeking them in the phonocardiogram and the A.D.C. disturbs the patient less than eliciting them from the pressure pulses of the left atrium and ventricle, and has an equally rational basis.

Genesis of the Third Heart Sound. In all varieties of mitral disease, irrespective of whether the lesion impedes or enhances ventricular filling, a particular event takes place 0.1 to $0.17 \mathrm{sec}$. after the closure of the aortic valve. This event is manifest in the sudden deceleration of the left atrial ' $y$ ' descent at the annular ascent point, and in the sudden reduction in the rate of outward movement of the left ventricular apex at the third sound point. The observation of the heart at operation and published work (Radner, 1957; Rushmer, 1961) suggest that the event is the vigorous early diastolic elongation of the left ventricle. It is shown in this study that the event occurs synchronously with the third heart sound, with the onset or waxing of the diastolic murmur of incompetence, and with the inward movement of the left ventricular apex when a third sound is present.

These factors are consistent with the following theory: during the cardiac cycle the apex of the left ventricle alters little in position. In systole ventricular contraction moves the atrio-ventricular ring towards the apex of the heart, and in early diastole vigorous elongation of the ventricles causes the base suddenly to ascend (Radner, 1957; Rushmer, 1961). When the left ventricle is distended with blood the distance from the base to the apex increases, but the mitral cusps and chordae do not elongate. Consequently, when the ventricle is distended beyond a certain point, vigorous elongation and ascent of the annulus fibrosus suddenly tenses the mitral cusps and chordae and produces noise, the third heart sound: traction on the cusps and chordae pulls inwards the apex of the left ventricle, the movement recorded on the A.D.C. at the time of the third sound. The anatomical arrangement of the chordae tendineae ensures that their tautening reduces the mitral orifice, and causes a degree of obstruction to atrio-ventricular flow. The sudden onset of a degree of obstruction checks ventricular filling, and reduces the rate of ' $y$ ' descent and the rate of outward movement of the left ventricular apex. When the left atrium still contains an excessive amount of blood under high pressure the sudden check causes the onset or waxing of the diastolic murmur.

Potain considered that "the dilating ventricle quickly reaches a point where the fibrous resistance of its wall limits its distension, and the latter, sharply arrested, causes a tension, a shock, and the gallop sound" (quoted from Dock et al., 1955). This study has shown that left ventricular filling 
and stasis are not achieved at the time of the third heart sound in severe mitral incompetence, but occur considerably later, at the end of the slow filling phase that is marked by an abnormally large left ventricular filling pressure gradient and a diastolic murmur. Our findings are evidence against Potain's concept, and support Dock's (1949) contention that in life the left ventricle does not tauten like a blown-up paper bag, but is capable of distending far beyond the size at which third sounds occur. These points, and other work relating to the causation of the third heart sound, have been reviewed recently (Nixon, 1961c).

\section{SUMMARY}

Left heart pressure pulses, phonocardiograms, and displacement curves of the left ventricular apex were recorded in patients with mitral valvular disease, and correlated with the findings at operation. Various patterns of left ventricular filling and stasis were recognized, and each was considered to be diagnostic of a particular valvular lesion.

A prolonged phase of filling that began with the third heart sound was present in every case of severe mitral incompetence. The belief that the third heart sound is caused by the left ventricle suddenly reaching the limit of its distension is not consistent with this finding.

At the time at which the left atrial annular ascent point was recorded, it was noted that the third heart sound occurred; the apex of the left ventricle moved inwards if a third heart sound was present; the ' $y$ ' descent of the left atrial pressure and the outward movement of the left ventricular apex were suddenly slowed; and the diastolic murmur of mitral incompetence made its appearance or increased suddenly in loudness. A theory that accommodates these findings is presented.

The authors are grateful to Mr. R. Addyman, Mr. H. Ketteringham, and Mrs. J. Blake for assistance, and thank the Board of Governors of the United Leeds Hospitals, the Nuffield Foundation, the Medical Research Council, and private benefactors for providing equipment. Transeptal left heart catheterization was learned in the Clinic of Surgery in the National Heart Institute, Bethesda, Maryland, and the technique for recording displacement curves of the left ventricular apex was learned in the Academisch Ziekenhuis, Utrecht, Holland: the authors wish to thank Dr. A. G. Morrow and Professor R. L. J. van Ruyven for their kindness in making this possible.

\section{REFERENCES}

Braunwald, E., Moscovitz, H. L., Amram, S. S., Lasser, R. P., Sapin, S. O., Himmelstein, A., Ravitch, M. M., and Gordon, A. J. (1955). The hemodynamics of the left side of the heart as studied by simultaneous left atrial, left ventricular, and aortic pressures; particular reference to mitral stenosis. Circulation, $12,69$.

Brock, R., Milstein, B. B., and Ross, D. N. (1956). Percutaneous left ventricular puncture in the assessment of aortic stenosis. Thorax, 11, 163.

Dock, W. (1949). The genesis of heart sounds. New Engl. J. Med., 241, 767.

(1959). The forces needed to evoke sounds from cardiac tissues, and the attenuation of heart sounds. Circulation, 19, 376.

—, Grandell, F., and Taubman, F. (1955). The physiologic third heart sound: its mechanism and relation to protodiastolic gallop. Amer. Heart J., 50, 449.

Gibson, A. G. (1907). The significance of a hitherto undescribed wave in the jugular pulse. Lancet, $2,1380$.

Henderson, Y. (1906). The volume curves of the ventricles of the mammalian heart. Amer. J. Physiol., 16, 325.

Hirschfelder, A. D. (1907). Some variations in the form of the venous pulse. Johns Hopk. Hosp. Bull., 18, 265.

(1910). Diseases of the Heart and Aorta. Lippincott, Philadelphia and London.

Leatham, A. (1952). Phonocardiography. Brit. med. Bull., 8, 334.

Mackenzie, J. (1902). The Study of the Pulse, Arterial, Venous, and Hepatic and of the Movements of the Heart. Young J. Pentland, Edinburgh and London.

Nixon, P. G. F. (1960). The transeptal approach to the left atrium in mitral regurgitation. Thorax, $15,225$. (1961a). The low-flow and the high-flow mitral diastolic murmurs. Brit. Heart J., 23, 462.

(1961b). Time relationships of the left atrial V wave in mitral valvular disease. Brit. Heart J., $23,637$.

(1961c). The third heart sound in mitral regurgitation. Brit. Heart J., 23, 677.

- , and Polis, O. (1962). The left atrial X descent. Brit. Heart J., 24, 173.

- , and Wooler, G. H. (1961). Rapid left ventricular filling and stasis in mitral regurgitation. Brit. Heart J., 23, 161 .

- - (1963). The left ventricular filling pressure gradient in mitral incompetence. Brit. Heart J., $25,382$.

$\longrightarrow$, and Radigan, L. R. (1960). The opening snap in mitral incompetence. Brit. Heart J., $22,395$.

Oakley, C., Yusuf, R., and Hollman, A. (1961). Coronary embolism and angina in mitral stenosis. Brit. Heart J., 23, 357.

Radner, S. (1957). Left atrial pressure curve: significance of annular ascent wing. Acta med. scand., $159,219$. 
Rihl, J. (1904). Experimentelle Analyse des Venenpulses bei den durch Extrasystolen verursachten UnregelmässigKeiten des Säugethierherzens. Z. exp. Path. Ther., 1, 43. Quoted by Wiggers, C. J. (1923). In Modern Aspects of the Circulation in Health and Diseases, 2nd ed., Chap. 12. Lea and Febiger, Philadelphia and New York.

Ross, J. (1959). Catheterization of the left heart through the interatrial septum: a new technique and its experimental evaluation. Surg. Forum, 1958, 9, 297.

Rushmer, R. F. (1961). Cardiovascular Dynamics, 2nd ed. Saunders, Philadelphia and London.

Sarnoff, S. J., Gilmore, J. P., and Mitchell, J. H. (1962). Influence of atrial contraction and relaxation on closure of mitral valve. Circulat. Res., 11, 26.

Schneider, H., and Klunhaar, E. W. J. M. (1961). Precordial low-frequency displacements of the thoracic wall. Method of recording and registration. Amer. Heart J., 61, 670.

Thayer, W. S. (1909). Further observations on the third heart sound. Arch. intern. Med., 4, 297.

Wiggers, C. J. (1921). Studies on the consecutive phases of the cardiac cycle. Amer. J. Physiol., 56, 415.

Wooler, G. H., Nixon, P. G. F., Grimshaw, V. A., and Watson, D. A. (1962). Experiences with the repair of the mitral valve in mitral incompetence. Thorax, 17, 49 . 